\title{
General relativity in R: visual representation of Schwarzschild space using different coordinate systems
}

\author{
Robin K. S. Hankin ${ }^{1}$ \\ 1 Auckland University of Technology
}

DOI: $10.21105 /$ jose. 00091

\section{Software}

- Review ${ }^{\pi}$

- Repository u

- Archive ${ }^{\top}$

Submitted: 06 November 2019 Published: 25 May 2021

\section{License}

Authors of papers retain copyright and release the work under a Creative Commons Attribution 4.0 International License (CC BY 4.0).

\section{Introduction}

In general relativity, Schwarzschild coordinates for a black hole have desirable properties such as asymptotic matching with flat-space spherical coordinates; but other coordinate systems can be used which have other advantages such as removing the non-physical coordinate singularity at the event horizon. Following Schwarzschild's original publication in 1916 of his spherically symmetrical solution to the vacuum Einstein field equations, a variety of coordinate transformations have been described that highlight different features of the Schwarzschild metric. These include: Kruskal-Szekeres (Kruskal, 1960; Szekeres, 1960), Eddington-Finkelstein (Eddington, 1924; Finkelstein, 1958), Gullstrand-Painleve (Gullstrand, 1922; Painlevé, 1921), Lemaitre (Lemaître, 1933), and various Penrose transforms with or without a black hole (Hawking \& Ellis, 1973). These are described in many undergraduate GR textbooks such as Schutz (2009) and Carroll (2019).

The schwarzschild package is a suite of visualization routines for the coordinate systems mentioned above. It creates a large number of mathematically accurate diagrams using the $\mathrm{R}$ programming language.

\section{Statement of Need}

In the teaching of numerical subjects such as physics, mathematical accuracy is an important requirement for informative diagrams. However, many users of such diagrams will want the ability to customise or otherwise modify them. In computer terminology, one would need the source code as well as the final image and such source code is not currently available. The schwarzschild R package (R Core Team, 2019), available under the General Public License, fills this need.

The package is intended as a resource for lecturers of general relativity and it is envisaged that the diagrams be used as visual teaching aids for understanding the Schwarzschild metric. A number of camera-ready PDF diagrams of black holes using a range of coordinate systems are presented as examples of the software's functionality; the software is extensively configurable to users' requirements.

The software has been used in two general relativity teaching contexts: firstly, as resources for astrophysics lectures at Auckland University of Technology; and secondly to support the Trin Tragula YouTube channel General relativity step by step which, as of October 2020 has over 150000 views and 1200 subscribers. 


\section{Functionality and usage}

The schwarzschild package presents structured $\mathrm{R}$ code with extensive inline documentation as part of an educational resource package. The package creates mathematically accurate diagrams illustrating different aspects of physics near a spherically symmetric black hole. Physical processes such as null geodesics and freely falling objects near a Schwarzschild black hole are simulated in $\mathrm{R}$ and plotted as usable and accurate PDF images.

The package defines over twenty functions that plot diagrams of spacetime in the vicinity of a black hole. The principal such function would be schwarzschild(), which shows a spacetime diagram near a non-spinning stationary black hole using Schwarzschild coordinates but many other coordinate systems are available, including Kruskal-Szekeres (kruskal() and variants), Lemaître coordinates (lemaitre()) and others.

The code itself is maintainable, extensible, and makes the connection between physics and plotted diagram explicit. The package is written to behave well in the wider ecology of $\mathrm{R}$ software.

\section{Examples}

Two example images are shown below in low-resolution JPG format. All nineteen vectorized PDF images can be seen in the package vignette or online through Microsoft Sharepoint. 


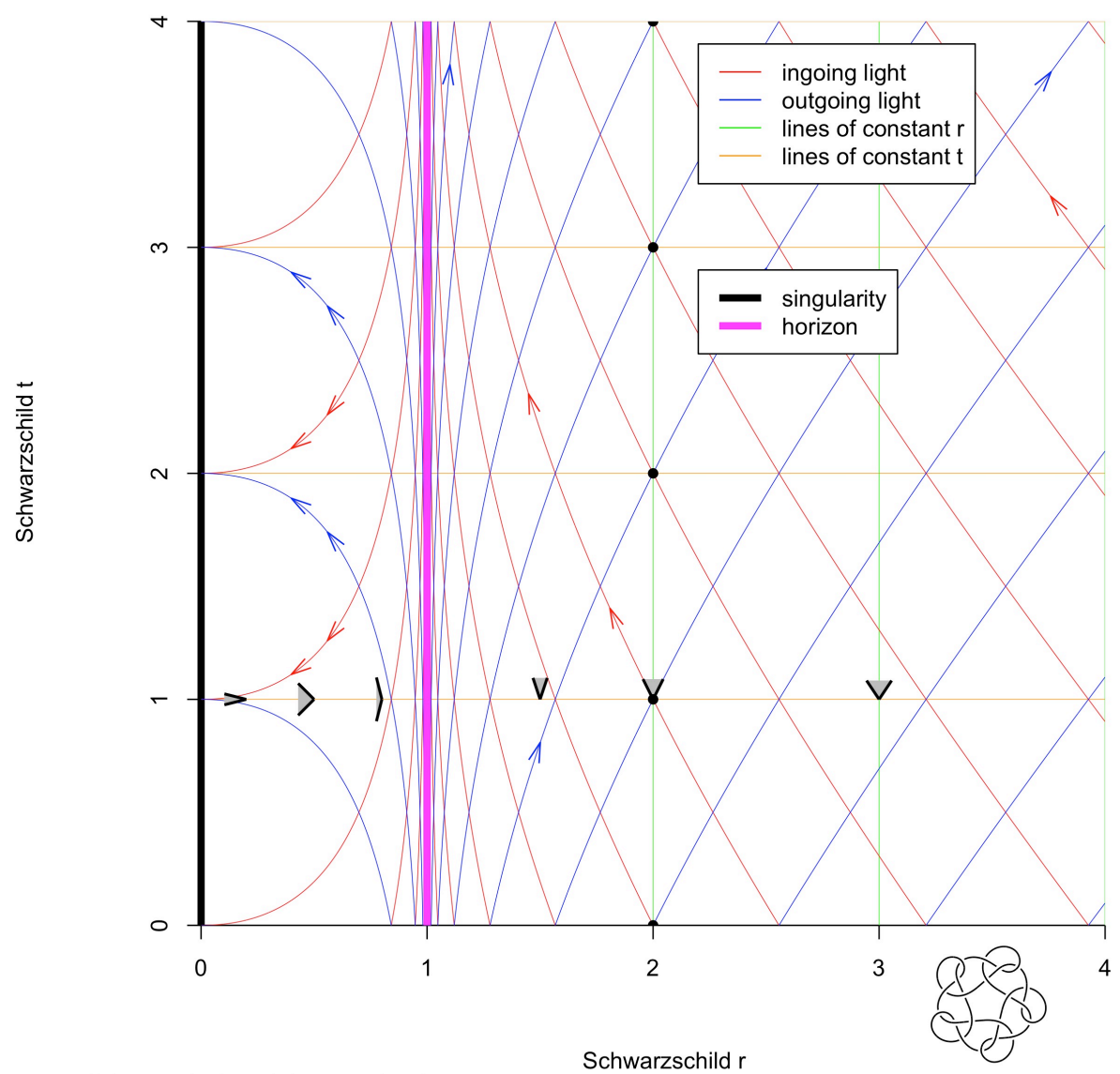

Schwarzschild $r$

Figure 1: Low resolution image of space near a black hole, using standard Schwarzschild coordinates. Neither ingoing light (red) nor outgoing light (blue) crosses the event horizon (fuschia); inside the black hole, ingoing light travels backwards in Schwarzschild time but nears the singularity. As the event horizon is approached from the outside, the light cones (gray) close up but as the event horizon is crossed, they become everted. As the singularity is approached, ingoing and outgoing light converge and meet the singularity horizontally 


\begin{tabular}{ll|}
$\square$ & leftward light \\
- & rightward light \\
$\square$ & lines of constant Schwarzschild $r$ \\
$\square$ & lines of constant Schwarzschild $\mathrm{t}$ \\
\hline & singularity \\
horizon
\end{tabular}

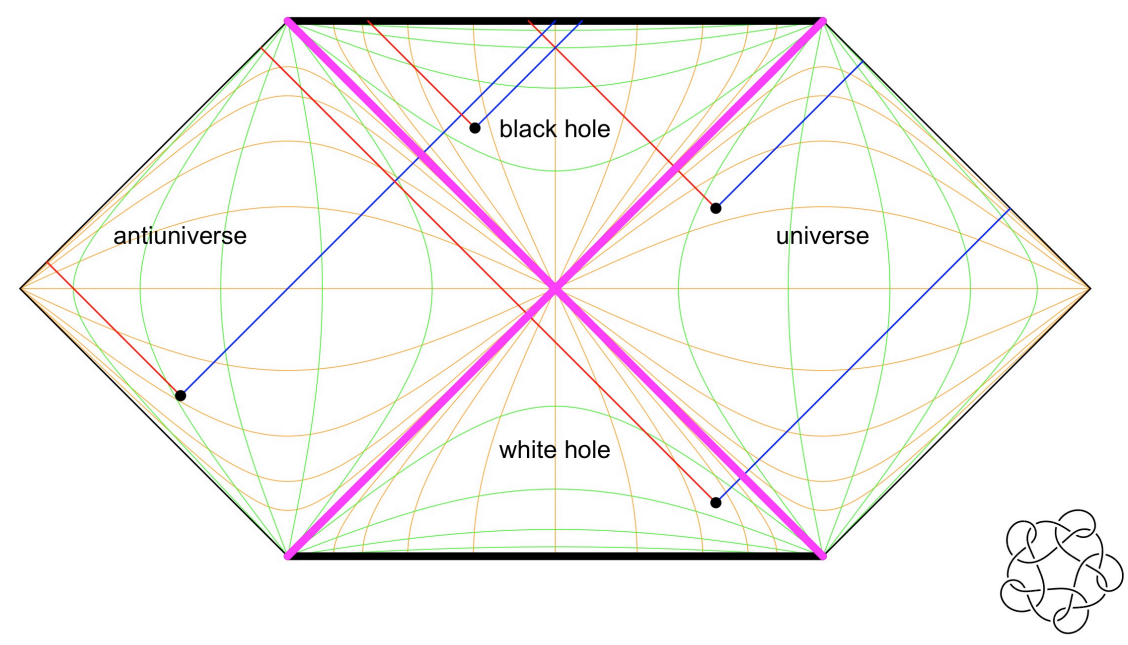

https://github.com/RobinHankin/schwarzschild.git

Figure 2: Low resolution image of maximally extended space near a black hole, using Kruskal-Szekeres coordinates. Ingoing light (red) and outgoing light (blue) are represented as $45^{\circ}$ lines. The event horizon (fuschia) is shown as a set of $45^{\circ}$ lines.

Neither ingoing nor outgoing light emitted from an object inside the event horizon can escape the black hole and the singularity (thick black line) is unavoidable; the area above the singularity is meaningless. Lines of constant Schwarzschild time are shown in orange and constant Schwarzschild radius in green; see how the lines of constant radius are spacelike, and lines of constant Schwarzschild time are timelike, inside the event horizon. The extended coordinates admit two new regions: an antiuniverse on the left and a white hole below the black hole. The only way that objects in the universe can have a causal link with objects in the antiuniverse is via the interior of the black hole and examples of intersecting null geodesics are shown. The white hole is a region from which escape is inevitable; outgoing null geodesics may emerge in either the universe or the antiuniverse

\section{References}

Carroll, S. M. (2019). Spacetime and geometry. Cambridge University Press.

Eddington, A. S. (1924). A comparison of Whitehead's and Einstein's formulae. Nature, $113(2832), 192$.

Finkelstein, D. (1958). Past-future asymmetry of the gravitational field of a point particle. Physical Review, 110(4), 965-967. 
Gullstrand, A. (1922). Allgemeine lösing des statischen einkörperproblems in der Einsteinschen gravitationstherorie. Arkiv för Matematik, Astronomi Och Fysik, 16(8), $1-15$.

Hawking, S. P., \& Ellis, G. F. R. (1973). The large scale structure of spacetime. Cambridge University Press.

Kruskal, M. D. (1960). Maximal extension of the Schwarzschild metric. Phys. Rev., 119, 1743.

Lemaître, G. (1933). The expanding universe. Soc. Sci. Bruxelles A, 53, 51.

Painlevé, M. P. (1921). La mécanique classique et la théorie de la relativité. Académie Des Sciences, 173, 677-680.

R Core Team. (2019). R: A language and environment for statistical computing. $\mathrm{R}$ Foundation for Statistical Computing. https://www.R-project.org/

Schutz, B. (2009). A first course in general relativity. Cambridge University Press.

Schwarzschild, K. (1916). Über das Gravitationsfeld eines Massenpunktes nach der Einsteinschen Theorie. Sitzungsberichte Der Königlich Preussischen Akademie Der Wissenschaften, 7, 189-196.

Szekeres, G. (1960). Publicationes Mathematicae Debrecen, 7, 285-301. 